\title{
Magnetic transition in NiPt alloy systems: experiment and theory
}

\author{
Uday Kumar $^{\mathrm{a}}$, K.G. Padmalekha ${ }^{\mathrm{b}}$, P.K. Mukhopadhyay ${ }^{\mathrm{a}}$, \\ Durga Paudyal $^{\mathrm{c}}$, Abhijit Mookerjee ${ }^{\mathrm{c}, *}$ \\ ${ }^{a}$ Laboratory for Condensed Matter Physics, S.N. Bose National Centre for Basic Sciences, JD Block, Sector 3, Salt Lake City, \\ Kolkata 700098, India \\ ${ }^{\mathrm{b}}$ Department of Physics, Indian Institute of Science, Bangalore 560012, India \\ ${ }^{c}$ Condensed Matter Theory Group, S.N. Bose National Centre for Basic Sciences, JD Block, Sector 3, Salt Lake City, \\ Kolkata 700098, India
}

\begin{abstract}
We report here the preparation and measurements on the susceptibility, sound velocity and internal friction for NiPt systems. We then compare these experimental results with the first principle theoretical predictions and show that there is reasonable agreement with experiment and theory.
\end{abstract}

Keywords: Sound velocity and attenuation; NiPt; Phase diagram

\section{Introduction}

The NiPt alloy system is an interesting study both because of earlier controversies about theo-

\footnotetext{
*Corresponding author. Tel.:+913323355706; fax: +913323353477 .

E-mail addresses: pkm@bose.res.in (P.K. Mukhopadhyay), dpaudyal@bose.res.in (D. Paudyal), abhijit@bose.res.in (A. Mookerjee).
}

retical predictions regarding its chemical stability and its magnetic properties.

Some initial studies predicted these systems to be phase separating contradicting experimental observations. In our previous work [1,2] we concluded that NiPt system is stable provided we take into account scalar relativistic corrections to the underlying Schrödinger equation and deal with both charge transfer and lattice relaxation effects properly.

For the $50 \%$ alloy there is also a disagreement regarding its magnetic properties. Early experiments 
indicated that disordered NiPt is ferromagnetic [3], while in the ordered phase it is paramagnetic [4]. Other authors found ordered NiPt to be antiferromagnetic [5]. Spin-polarized local-density approximation based calculations seem to indicate that even $\mathrm{Ni}_{25} \mathrm{Pt}_{75}$ shows some local magnetic moment, whereas experiments seem to indicate that there is no magnetism at all.

In this communication we report susceptibility, sound velocity and internal friction experiments on a series of $\mathrm{NiPt}$ alloys with $\mathrm{Pt}$ concentrations varying between $41 \%$ and $76 \%$. We have also carried out spin-polarized local-density-based tight-binding linearized muffin-tin orbitals (TBLMTO) calculations for the magnetic properties of these alloys and analyse the experimental results in this light.

\section{Experimental details}

\subsection{Sample preparation and characterization}

We made four different compositions of the alloy, $\mathrm{Ni}_{x} \mathrm{Pt}_{1-x}$ (in at\%). First an amount of $\mathrm{Ni}$ was cut from an ingot of pure Ni. After weighing it, a target amount of $\mathrm{Pt}$ was cut from pure $\mathrm{Pt}$ wire. Since there would always be a little bit of error in weight adjustments, the exact target composition was never reached, but we determined the final composition to be close to it. This is tabulated in Table 1.

The materials for the required composition were then put in an arc furnace and melted in a flowing argon atmosphere. After melting, the mass formed into a shinning button. We measured the maximum mass loss to be $0.8 \%$. These are now taken

Table 1

Composition analysis for the NiPt samples

\begin{tabular}{llll}
\hline $\begin{array}{l}\text { Target } \\
\text { composition }\end{array}$ & $\begin{array}{l}\text { Actual } \\
\text { composition }\end{array}$ & $\begin{array}{l}\text { Nearest whole } \\
\text { number } \\
\text { composition }\end{array}$ & $\begin{array}{l}\text { Lattice } \\
\text { constants in } \\
\mathrm{nm}\end{array}$ \\
\hline $\mathrm{Ni}_{60} \mathrm{Pt}_{40}$ & $\mathrm{Ni}_{58.7} \mathrm{Pt}_{41.3}$ & $\mathrm{Ni}_{59} \mathrm{Pt}_{41}$ & 0.372 \\
$\mathrm{Ni}_{50} \mathrm{Pt}_{50}$ & $\mathrm{Ni}_{49.6} \mathrm{Pt}_{50.4}$ & $\mathrm{Ni}_{50} \mathrm{Pt}_{50}$ & 0.376 \\
$\mathrm{Ni}_{45} \mathrm{Pt}_{55}$ & $\mathrm{Ni}_{44.6} \mathrm{Pt}_{55.4}$ & $\mathrm{Ni}_{45} \mathrm{Pt}_{55}$ & 0.377 \\
$\mathrm{Ni}_{25} \mathrm{Pt}_{75}$ & $\mathrm{Ni}_{23.9} \mathrm{Pt}_{76.1}$ & $\mathrm{Ni}_{24} \mathrm{Pt}_{76}$ & 0.384 \\
\hline
\end{tabular}

out and initial homogenizations were done under sealed and evacuated quartz ampoules at $1000^{\circ} \mathrm{C}$ for $12 \mathrm{~h}$. Then they were quenched to room temperature. Afterwards they were carefully cold rolled to about $0.5 \mathrm{~mm}$ thickness and cut into reed shapes.

The samples are then again put in evacuated quartz ampules and heat treated to $1000^{\circ} \mathrm{C}$ for $72 \mathrm{~h}$. After quenching, they were finally annealed at $200{ }^{\circ} \mathrm{C}$ for $4 \mathrm{~h}$ to remove the stresses due to thermal shock that develops due to fast quenching.

The crystal structures of the samples were then measured in a standard XRD instrument (Philips make). Scans were taken from $4^{\circ}$ to $90^{\circ}$ at an interval of $0.02^{\circ}$ with a step time of $0.5 \mathrm{~s}$. Diffractograms for the samples are shown in Fig. 1.

The analysis of the XRD data indicate that all the samples have signatures of the face centred cubic (FCC) structure. If the alloy was ordered, it would have showed the signatures of the L10, L12 or other relevant superstructures. We do not see any indication of that. This implies that all the samples were probably in the disordered phase which is expected because of homogenization at and quenching from $1000^{\circ} \mathrm{C}$. There is good agreement between our experimental lattice parameters and that of Parra and Cable [3].

Fig. 2 shows the lattice constants as a function of the Pt concentration. The figure clearly shows deviations from Vegard's Law and a positive 'bowing' effect. This is to be expected because of the large size mismatch between $\mathrm{Ni}$ and $\mathrm{Pt}$ atoms. The maximum deviation occurs at around the 50\% concentration.

\subsection{Susceptibility measurements}

In Fig. 3 we show the data for the susceptibilities of the four samples. All except for $\mathrm{Ni}_{59} \mathrm{Pt}_{41}$ were done till liquid helium temperature. The measurements were done in a standard double balanced coil technique. Driving field was about 100 Oe and frequency was $120 \mathrm{~Hz}$ for the three samples, whereas for $\mathrm{Ni}_{59} \mathrm{Pt}_{41}$ it was $33 \mathrm{~Hz}$. We determine the Curie temperature $T_{\mathrm{c}}$ as the temperature at which $\partial \chi / \partial T$ shows a maximum. The experimental error in the determination of susceptibility 


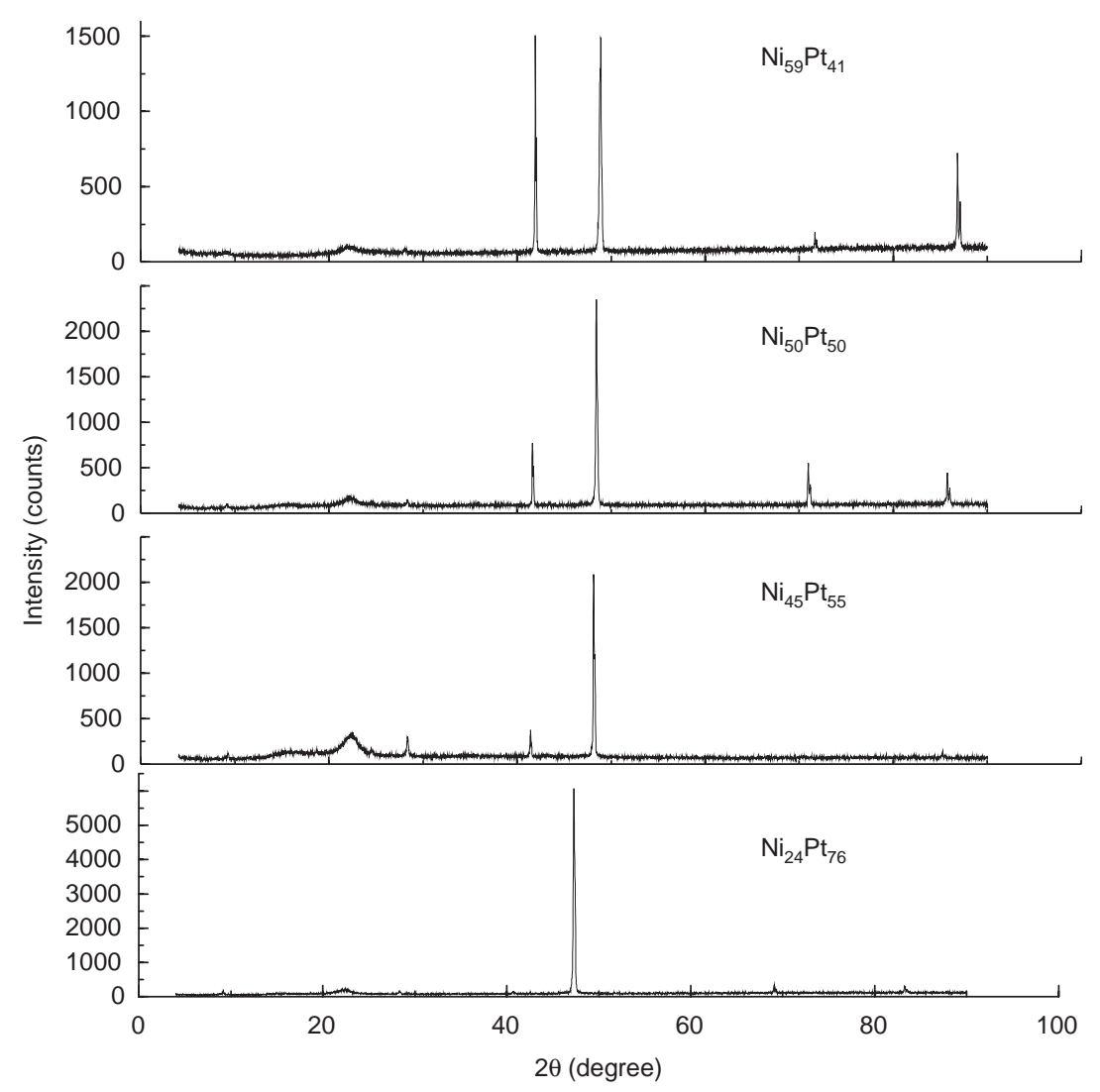

Fig. 1. X-ray diffractograms for NiPt alloys at four different compositions.

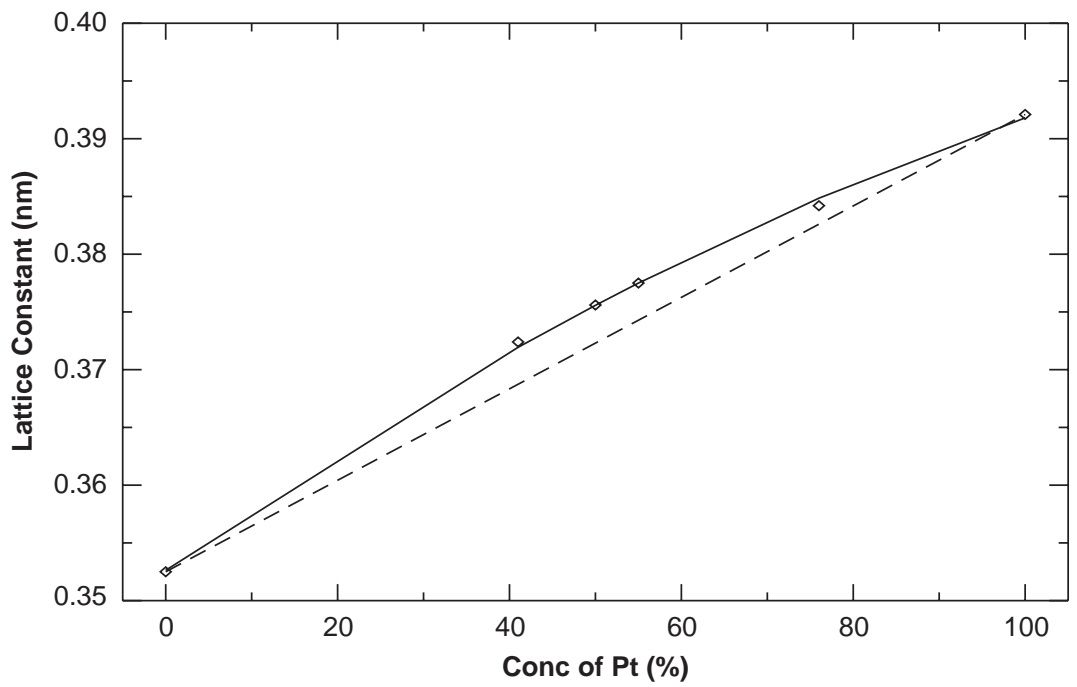

Fig. 2. Deviations between Vegard's Law (dashed line) and experimental data (diamond symbols). The smooth line is given as a guide to the eyes. 

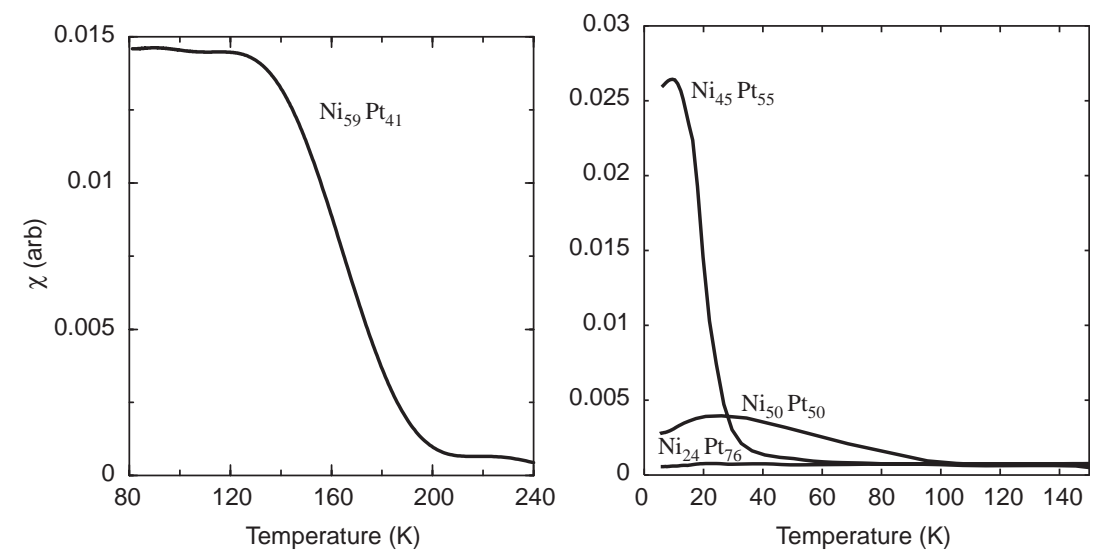

Fig. 3. Susceptibility vs. temperature curves for NiPt alloys with different compositions: (left) $41 \%$ Pt and (right) $50 \%$, $55 \%$ and $76 \% \mathrm{Pt}$

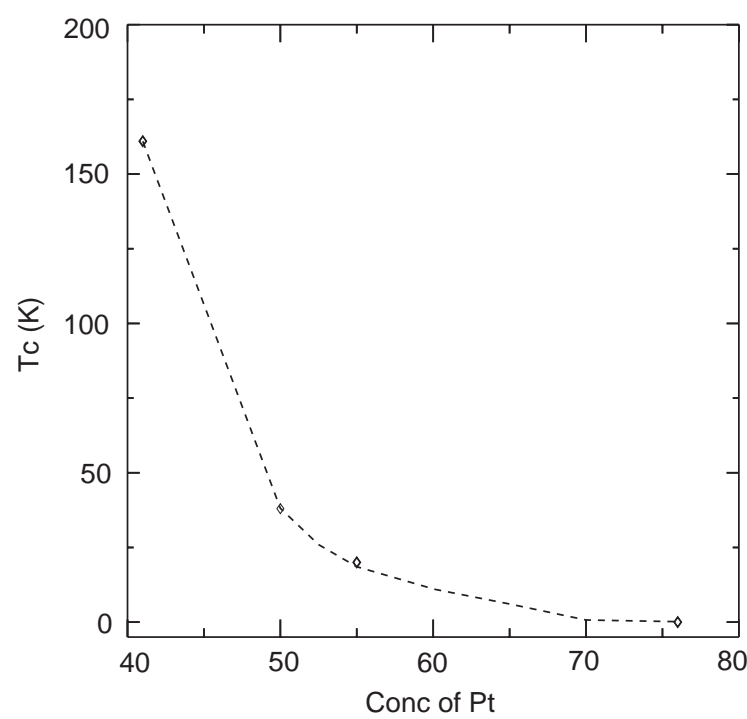

Fig. 4. Experimental Curie temperature (diamond symbols) vs. the alloy composition for NiPt alloys. Dashed line is a guide for the eyes.

is $\sim 0.01 \%$ and the error in the determination of temperature $(T)$ is about $\pm 0.5 \mathrm{~K}$. These errors contribute to the error in the determination of $T_{\mathrm{c}}$. The trend in the variation of $T_{\mathrm{c}}$ with alloy composition is shown in Fig. 4.

\subsection{Sound velocity and attenuation}

In this section we describe the sound velocity and internal friction $\left(Q^{-1}\right)$ measurements done on the systems as a function of temperature. However, since the present set-up does not reach below about $77 \mathrm{~K}$, data for only the $\mathrm{Ni}_{59} \mathrm{Pt}_{41}$ are shown in Fig. 5. The top figure shows the sound velocity results and the bottom one shows the internal friction data. In this case we could measure to temperature lower than $T_{\mathrm{c}}$. The precision in the measurement of $\delta V / V$ (where $V$ is the sound velocity and $\delta V$ is the change in it from a reference value) is about $50 \mathrm{ppm}$. As mentioned before the error in the measurement of $T$ is $\pm 0.05 \mathrm{~K}$. Finally, the error in the internal friction is slightly larger than $\sim 2 \%$.

These measurements are done by the vibrating reed technique. For this the sample was taken in the form of a reed and clamped at one end in the sample holder. The free end was made to resonate by electrostatic drive from one driver electrode placed near one surface there. Another electrode at the opposite side picked up this signal and fed to a lockin amplifier (LIA). This LIA was run at $2 \mathrm{f}$ mode from the reference provided by the signal generator for the driver. An integrator completed the phase locked loop that was set up to track the changes in resonance characteristics of the sample as the temperature was varied. The temperature was measured with the help of a platinum resistance thermometer. Detailed description of the vibrating reed technique can be found in Ref. [6].

From Fig. 5 (top), we see that as the temperature was lowered, initially the sound velocity was 

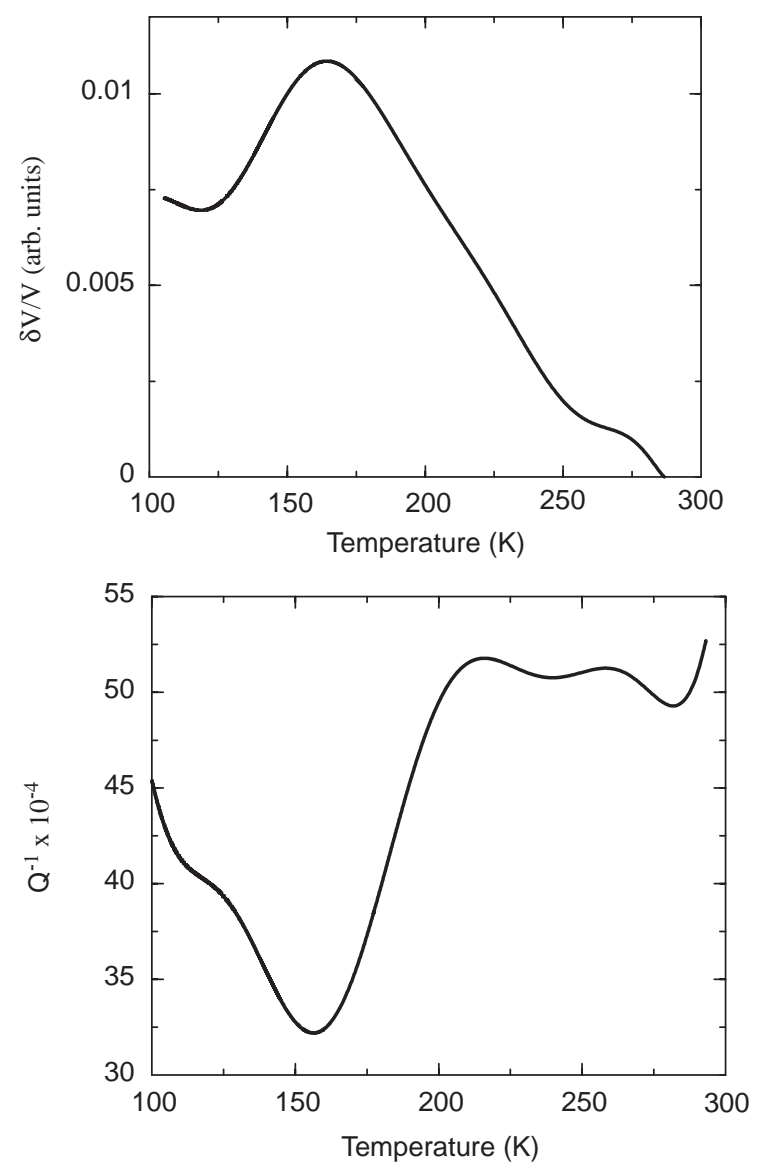

Fig. 5. Relative change in sound velocity (top) and internal friction vs. temperature for the alloy $\mathrm{Ni}_{59} \mathrm{Pt}_{41}$ (bottom).

increasing. However, as the temperature approached $T_{\mathrm{c}}$, the lattice softened up and the sound velocity showed a good amount of drop. However, for $T$ far below $T_{\mathrm{c}}$, the sound velocity again picked up, signaling the end of the magnetic transition and returned to the background lattice elastic limit. In case of internal friction (Fig. 5, bottom), we see an exactly complementary behaviour. Here the general lowering of $Q^{-1}$ was enhanced as $T$ approached $T_{\mathrm{c}}$, till it dropped to about $50 \%$ of the room temperature value. And just like the sound velocity, it tended to recover to a general lower value at still lower temperatures.

Both these elastic measurements show that with the onset of magnetic transition, the background lattice elastic properties get largely modified due to strong spin lattice coupling in this system. In case of sound velocity which is the real part of elasticity, the elastic modulus fell to about $50 \%$ across the transition. The peak temperature, $T_{\mathrm{p}}$, is about $161 \mathrm{~K}$ which is about the same as the $T_{\mathrm{c}}$ as determined from the real part of $\chi$. However, it is well known that the effect of ferromagnetism on spin lattice coupling can start from a little below $2 T_{\mathrm{c}}[7,8]$, we actually see that the sound velocity started a faster rise below around $260 \mathrm{~K}$. The same argument predicts reduction of effect of magnetic transition down from $T_{\mathrm{c}} / 2$ and it is also seen in the recovery of sound velocity.

In the similar vein, we can see the effect of magnetic transition on $Q^{-1}$ data too. We see that the general fall of $Q^{-1}$ was arrested below $250 \mathrm{~K}$ and a massive fall in it occurred. The minimum temperature, $T_{\mathrm{m}}$, is about $158 \mathrm{~K}$-below $T_{\mathrm{p}}$. Again this is well documented [7] that $T_{\mathrm{m}}$ (determined from the imaginary part) always lags behind $T_{\mathrm{p}}$ (determined from the real part). And just like the real part, $Q^{-1}$ also recovered from the effects of magnetic transition below about $T_{\mathrm{c}} / 2$. Hence we find that the effect of magnetic transition was to both soften the lattice and also reduce the anharmonicity of it simultaneously. We could have tried to fit a general elastic background to the data and extract the magnetic part from it. However, there are major problems in it. We need to have data from much above $2 T_{\mathrm{c}}$ to much below $T_{\mathrm{c}} / 2-$ which are not available. And in any case the fittings are always ambiguous and subject to intense debate. So we would not like to do it here.

\section{Theoretical and computational methods}

For a theoretical estimation of the Curie temperatures we have followed two different approaches. In the first method, we calculate the magnetic pair energies and then use the mean-field approximation to evaluate the approximate Curie temperatures. For magnetic pair interaction energies we embed different atoms with their accompanying spins in an averaged non-magnetic disordered medium at two different sites a distance 
$R$ apart. The pair interactions are defined as

$J_{\mathrm{AA}}(R)=E_{\mathrm{AA}}^{\uparrow \uparrow}+E_{\mathrm{AA}}^{\downarrow \downarrow}-E_{\mathrm{AA}}^{\uparrow \downarrow}-E_{\mathrm{AA}}^{\downarrow \uparrow}$,

$J_{\mathrm{BB}}(R)=E_{\mathrm{BB}}^{\uparrow \uparrow}+E_{\mathrm{BB}}^{\downarrow \downarrow}-E_{\mathrm{BB}}^{\uparrow \downarrow}-E_{\mathrm{BB}}^{\downarrow \uparrow}$,

$J_{\mathrm{AB}}(R)=E_{\mathrm{AB}}^{\uparrow \uparrow}+E_{\mathrm{AB}}^{\downarrow \downarrow}-E_{\mathrm{AB}}^{\uparrow \downarrow}-E_{\mathrm{BB}}^{\downarrow \uparrow}$.

We obtain these pair interactions directly using the orbital peeling technique [9] in conjunction with the augmented space recursion [10] introduced by us earlier in a tight-binding linearized muffin-tin orbitals basis (TB-LMTO) [11].

Using these interaction energies the BraggWilliams mean-field expression for Curie temperature is

$T_{\mathrm{c}}=\frac{1}{k_{\mathrm{B}}}\left[-x^{2} J_{\mathrm{AA}}-(1-x)^{2} J_{\mathrm{BB}}+2 x(1-x) J_{\mathrm{AB}}\right]$

$J_{\mathrm{AA}}=\sum_{R} J_{\mathrm{AA}}(R)$, etc.

The other approach is the Mohn-Wohlfarth (MW) procedure [12]:

$$
\left(\frac{T_{\mathrm{c}}}{T_{\mathrm{S}}}\right)^{2}+\frac{T_{\mathrm{c}}}{T_{\mathrm{SF}}}-1=0,
$$

where $T_{\mathrm{S}}$ is the Stoner temperature calculated from the relation

$\left\langle I\left(E_{\mathrm{F}}\right)\right\rangle \int_{-\infty}^{\infty} \mathrm{d} E N(E)\left(\frac{\partial f}{\partial E}\right)=1$

$\left\langle I\left(E_{\mathrm{F}}\right)\right\rangle$ is the concentration averaged Stoner parameter, $N(E)$ is the density of states per atom per spin of the paramagnetic state [13] and $f(E)$ is the Fermi distribution function. The spin fluctuation temperature $T_{\mathrm{SF}}$ is given by

$T_{\mathrm{SF}}=\frac{m^{2}}{10 k_{\mathrm{B}}\left\langle\chi_{0}\right\rangle}$

$\left\langle\chi_{0}\right\rangle$ is the concentration weighted exchange enhanced spin susceptibility at equilibrium and $m$ is the averaged magnetic moment per atom. $\chi_{0}$ is calculated using the relation by Mohn and Wohlfarth [12] and Gersdorf [14]:

$\chi_{0}^{-1}=\frac{1}{2 \mu_{\mathrm{B}}^{2}}\left(\frac{1}{2 N^{\uparrow}\left(E_{\mathrm{F}}\right)}+\frac{1}{2 N^{\downarrow}\left(E_{\mathrm{F}}\right)}-I\right)$

$N^{\uparrow}\left(E_{\mathrm{F}}\right)$ and $N^{\downarrow}\left(E_{\mathrm{F}}\right)$ are the spin-up and spindown partial density of states per atom at the Fermi level for each species in the alloy.

\subsection{Lattice structure}

As shown in Table 2 and in comparison with Table 1, our measured values of lattice parameters match well with the previously measured lattice parameters. In all concentrations we have got the FCC structures as par with the previous experiment by Parra and Cable [3]. It should be noted that our calculations show that for the ordered $50 \%$ alloy, the structure with a small tetragonal distortion has the lowest energy. The experimental lack of signature of any tetragonal distortion for the $50 \%$ alloy further strengthens our belief that the alloy was indeed disordered.

\subsection{Curie temperature}

The experimental measurements in NiPt alloys with $40 \%, 50 \%$ and $55 \%$ concentration of Pt show the ferromagnetic to paramagnetic transitions are at 161,38 and $20 \mathrm{~K}$.

Experimentally we find that the alloys up to $55 \% \mathrm{Pt}$ do show ferromagnetic behaviour, certainly in the disordered phase. However, at $76 \%$ of $\mathrm{Pt}$ the alloy becomes non-magnetic. Our first principles calculations agree with this up to $55 \%$ of Pt. However, our theoretical calculations indicate that ferromagnetism persists even at $75 \%$ of $\mathrm{Pt}$. We are unable to explain why this should be and why the Stoner-like arguments fail in these compositions. Table 3 shows that the Mohn-Wohlfarth and Bragg-Williams approaches both give qualitatively the correct trend in $T_{\mathrm{c}}$ up to $55 \%$ of Pt. Both approaches show the $50 \%$ alloy to be ferromagnetic, settling an earlier dispute. It is interesting to note that if we do not take into account local lattice distortion due to size mismatch in the NiPt alloys, the 50\% alloy yields a

Table 2

Equilibrium lattice constants in $\mathrm{nm}$

\begin{tabular}{ll}
\hline Alloy composition & Lattice constant $(\mathrm{nm})$ \\
\hline $\mathrm{Ni}_{60} \mathrm{Pt}_{40}$ & 0.372 \\
$\mathrm{Ni}_{50} \mathrm{Pt}_{50}$ & 0.376 \\
$\mathrm{Ni}_{45} \mathrm{Pt}_{55}$ & 0.378 \\
$\mathrm{Ni}_{25} \mathrm{Pt}_{75}$ & 0.384 \\
\hline
\end{tabular}


Table 3

Curie temperatures in NiPt alloys

\begin{tabular}{llll}
\hline Composition & Experiment & $\begin{array}{l}\text { Theory I } \\
\text { (Mohn- } \\
\text { Wohlfarth) }\end{array}$ & $\begin{array}{l}\text { Theory II } \\
\text { (Bragg- } \\
\text { Williams) }\end{array}$ \\
\hline $\mathrm{Ni}_{60} \mathrm{Pt}_{40}$ & 161 & 125 & 232 \\
$\mathrm{Ni}_{50} \mathrm{Pt}_{50}$ & 38 & 38 & 40 \\
$\mathrm{Ni}_{45} \mathrm{Pt}_{55}$ & 20 & 17 & 35 \\
\hline
\end{tabular}

Curie temperature of $76 \mathrm{~K}$ which is quantitatively at variance with experiment. We have included size mismatch effects within our TB-LMTO-Augmented Space Recursion.

\section{Conclusions}

In this communication we have reported structural and magnetic properties of NiPt alloys. The structure was probed by XRD experiments, while the magnetic properties were probed by susceptibility, sound velocity and attenuation. Our experimental findings of lattice parameters agree with earlier experiments and confirm that our samples were in the disordered state. We have also carried out a theoretical study of the same alloy system and compared the theoretical predictions with the experimental results. The theory correctly predicts the trend in the Curie temperature. The $\mathrm{Ni}_{50} \mathrm{Pt}_{50}$ alloy was found to be ferromagnetic, settling and earlier dispute. Our theory cannot explain why ferromagnetism disappears around the $\mathrm{Ni}_{25} \mathrm{Pt}_{75}$ composition.

\section{Acknowledgements}

We would like to thank Drs. A. Mitra and P.K. De for the samples, Prof. A.K. Majumder for the use of his arc furnace and Prof. A.K. Raychaudhuri for measurement till $5 \mathrm{~K}$. We would also like to thank Dr. D. Das for his various helps at critical stages and Mr. S. Chanda for help in rolling the samples. We would like to thank Mr. D. Hazra for his help with XRD analysis. We would like to acknowledge partial support from TWAS under its project grant 97-212 RG/PHYS/AS and the CSIR project grant 03(948)/02-EMR-II.

\section{References}

[1] D. Paudyal, T. Saha-Dasgupta, A. Mookerjee, J. Phys. Condens. Matter 15 (2003) 1029.

[2] D. Paudyal, T. Saha-Dasgupta, A. Mookerjee, J. Phys. Condens. Matter 16 (2004) 2317.

[3] R.E. Parra, J.W. Cable, Phys. Rev. B 21 (1980) 5494.

[4] C.E. Dahmani, M.C. Cadeville, J.M. Sanchez, J.L. MoranLopez, Phys. Rev. Lett. 55 (1985) 1208.

[5] J.B. Staunton, B.L. Gyorffy, D.D. Johnson, F.J. Pinski, G.M. Stocks, in: V. Kumar, O.K. Andersen, A. Mookerjee (Eds.), Proceedings of Miniworkshop on electronic structure calculations and working group on disordered alloys, World Scientific, Singapore, 1994.

[6] P.K. Mukhopadhyay, A.K. Raychaudhuri, J. Phys. E 20 (1987) 507.

[7] P.K. Mukhopadhyay, A.K. Raychaudhuri, J. Phys. C 21 (1988) L385.

[8] P.K. Mukhopadhyay, A.K. Raychaudhuri, J. Appl. Phys. 67 (1990) 5235.

[9] N.R. Burke, Surf. Sci. 58 (1976) 349.

[10] T. Saha, I. Dasgupta, A. Mookerjee, Phys. Rev. B 50 (1994) 13267.

[11] O.K. Andersen, O. Jepsen, Phys. Rev. Lett. 53 (1984) 2581.

[12] P.H. Mohn, E.P. Wohlfarth, J. Phys. F 17 (1987) 2421.

[13] O. Gunnarsson, J. Phys. F 6 (1976) 587.

[14] R. Gersdorf, J. Phys. Radium 23 (1962) 726. 